Review Article

\title{
Mechanisms Underlying the Prevention and Treatment of Cholelithiasis Using Traditional Chinese Medicine
}

\author{
Qiliang Chen, ${ }^{1}$ Yuanyuan Zhang, ${ }^{1}$ Shunan Li, ${ }^{1}$ Shujiao Chen, \\ Xuejuan Lin, ${ }^{1}$ Candong Li $(1),{ }^{1}$ and Tetsuya Asakawa $\circledast^{1,2}$ \\ ${ }^{1}$ Research Base of Traditional Chinese Medicine Syndrome, Fujian University of Traditional Chinese Medicine, Fuzhou 350122, China \\ ${ }^{2}$ Department of Neurosurgery, Hamamatsu University School of Medicine, Handayama, Hamamatsu-City, Shizuoka, Japan
}

Correspondence should be addressed to Candong Li; fjzylcd@126.com and Tetsuya Asakawa; asakawat1971@gmail.com

Received 19 March 2019; Revised 17 May 2019; Accepted 2 June 2019; Published 17 June 2019

Academic Editor: José L. Rios

Copyright (C) 2019 Qiliang Chen et al. This is an open access article distributed under the Creative Commons Attribution License, which permits unrestricted use, distribution, and reproduction in any medium, provided the original work is properly cited.

Cholelithiasis is a major public health concern that necessitates highly effective, feasible, and recurrence-preventing therapies. Currently available surgical treatments and medications cannot effectively avoid the recurrence of cholelithiasis. Hence, several Chinese herbal compounds (CHCs) are considered for the treatment of cholelithiasis, considering that they can effectively discharge gallstones and prevent the recurrence of such condition. In the present narrative review, we aim to summarize the underlying mechanisms of currently used CHCs in the treatment of cholelithiasis and to describe the current situation of traditional Chinese medicine (TCM) use for cholelithiasis. Several commonly used CHCs were used to illustrate these issues. We found that the mechanisms underlying the $\mathrm{CHC}$ treatments rely on the amelioration of the biliary dynamics factors, maintenance and protection of the liver function, reduction of the cholesterol and bilirubin levels, and regulation of the inflammatory reactions. CHCs as treatments based on TCM can ameliorate the overall bodily function, thereby preventing the recurrence of cholelithiasis. Appropriate application of CHCs would be beneficial for patients and clinicians, although the safety and efficacy of CHCs need further verification.

\section{Introduction}

Cholelithiasis is a common biliary tract disease with high morbidity of approximately $10 \%-15 \%$ [1]. The most dominant symptom of cholelithiasis is severe abdominal pain accompanied with chills, fever, and jaundice recurrence; these symptoms substantially impact the quality of life (QOL) of the patients. Notably, only $<20 \%$ of patients with cholelithiasis develop evident symptoms, whereas the remaining, particularly those with mild cholelithiasis, are typically asymptomatic [2]. However, the absence of symptoms does not indicate fitness. When the pathophysiological factors are not suppressed in asymptomatic patients, the disease may progress, resulting in serious consequences. Hence, cholelithiasis is a major public health concern that necessitates highly effective, feasible, and recurrence-preventing therapies. However, the recurrence of cholelithiasis is a crucial concern for the existing treatments, which is attributable to the complex pathogenic mechanisms of cholelithiasis.
Cholelithiasis can be anatomically classified as gallstones, intrahepatic bile duct stones, and extrahepatic bile duct stones, and it can be chemically classified as cholesterol stones, pigmented stones, and mixed stones. Different types of cholelithiasis may exhibit different pathogenesis. Typically, the development of cholelithiasis is closely associated with impairments in the metabolism of bile. The most predominant impairments are abnormal bilirubin and lecithin levels. Ahmed et al. confirmed that serum bilirubin may bind with free metal ions, particularly calcium ions, thereby forming relevant bilirubinate [3]. Conversely, lecithin polymerizes the insoluble microparticles with bile acid during bile secretion [4]. These insoluble products can cause gallstone formation. Moreover, cholelithiasis in advanced stages is occasionally accompanied with complications that may affect the pathophysiology of cholelithiasis. For example, gallstones stimulate the gallbladder mucosa, leading to chronic inflammation and acute secondary infection of the gallbladder [5]. Intrahepatic bile duct stones are associated with the occurrence 
of intrahepatic cholangiocarcinoma [6]. These complicated diseases may further deteriorate the QOL and lead to a poor treatment outcome. Therefore, making an appropriate diagnosis and subsequent effective treatments during the early stages, particularly for asymptomatic patients, is crucial to improve the clinical outcome of patients with cholelithiasis.

Nowadays, both surgical and nonsurgical treatments are available for cholelithiasis. The surgical treatments, namely, lithotripsy and laparotomy, are rarely used for cholelithiasis treatment. Traditionally, laparoscopic cholecystectomy (LC) is the gold standard treatment for symptomatic gallstones. However, LC includes potential surgical risks, such as biliary bleeding, bile duct stricture, common-bile-duct recurrence stones, and postcholecystectomy syndrome, and it may also increase the incidence of colon cancer [7]. Endoscopic retrograde cholangiopancreatography (ERCP), which was first reported in 1974, has been used as a standard treatment for bile duct stones. Most bile duct stones $(>90 \%)$ can be diagnosed and removed by any of the following techniques: ERCP, endoscopic sphincterotomy, or extraction balloon trawl [8]. However, ERCP has several limitations. It is an invasive surgery that may induce complications, such as cholecystitis and pancreatitis [9]. Treatment using ERCP is challenging in cases of large bile duct stones (>15 mm diameter) [10] and surgically altered anatomy [11]. Combining ERCP and LC in the same surgical session has been suggested to reduce possible conversions and complications [9]. Nakai et al. reported that, under endoscopic ultrasound guidance, enteroscopy-assisted ERCP is the potential technique for endoscopic management of bile duct stones in patients with surgically altered anatomy [11]. With the development of endoscopic technology, laparoscopic cholecystolithotomy with gallbladder preservation (LCGP) has been proposed by numerous clinicians because LCGP preserves gallbladder function, is minimally invasive, causes less surgical injury, and results in better QOL [12]. However, the efficacy of LCGP is controversial. The primary concerns toward LCGP are the efficacy of stone removal and its recurrence rate. In addition, the relatively strict indications of LCGP, such as gallbladder contraction function, gallbladder wall thickness, and gallstone characteristics (location, size, and number), limit the long-term use of LCGP. Meanwhile, the most commonly used medications for cholelithiasis are litholytic drugs, such as deoxycholic acid. However, the use of these medications is limited by expensive cost and noticeable adverse events, such as the development of hepatotoxicity and atherosclerosis [13].

Recurrence of gallstone formation is the major challenge for both clinicians and patients. None of the aforementioned treatments, including LC, ERCP, LCGP, surgical treatment, medications, and even complete gallbladder removal, can effectively prevent the recurrence of bile duct stones. Hence, several Chinese herbal compounds (CHCs) are considered for cholelithiasis treatment because they can effectively discharge gallstones [14] and prevent such recurrence [1522]. Additionally, noninvasive traditional Chinese medicines (TCM) are easily accepted by asymptomatic patients who may potentially refuse surgical treatments. However, a systematic review in 2013 showed that the Chinese herbs such as Qingdan, Danshu, Paishi, and Rongdanpaishi capsules are not efficacious for cholelithiasis treatment. However, the studies included in this review are of poor quality, thereby limiting the worthiness of this published evidence [23]. Currently, several novel CHCs have been developed. The present narrative review aims to summarize the underlying mechanisms of currently used CHCs in the treatment of cholelithiasis and describe the current situation of TCM use for cholelithiasis.

\section{Current Situation of TCM Use for Cholelithiasis}

TCM has been used to treat cholelithiasis for centuries. The knowledge on TCM in relation to cholelithiasis was obtained by studying the autopsy and observing the discharged stones in ancient China, when modern diagnostic equipment was not yet available. All TCM therapies, including acupuncture and CHCs, were selected based on the TCM syndromes.

Acupuncture is often employed to relieve pain in patients. It is predominantly effective in alleviating biliary spasm and pain [24]. The common acupoints for treating cholelithiasis are the gallbladder, liver, and bladder meridians, such as Danshu (BL19), Riyue (GB24), and Qiuxu (GB40). However, studies indicating the benefit of acupuncture in discharging gallstones, attributed to its strengthening effect on the gallbladder contraction and bile secretion, are limited [25-27].

$\mathrm{CHCs}$ have been used for treating lithiasis, including cholelithiasis and kidney stones. Selection of the CHCs is based on the principles of syndrome differentiation treatment. In TCM, cholelithiasis is divided into the following five types according to the TCM syndromes: (1) Liver depression and qi stagnation (syndrome of stagnation of the liver and qi), (2) Wetness heat of liver and gallbladder (syndrome of dampness-heat of the liver and gallbladder), (3) Liver yin deficiency (liver yin deficiency syndrome), (4) Blood stasis stagnation (blood stasis syndrome), and (5) Heat-toxic stagnation. The newest guidelines for using $\mathrm{CHCs}$ are as follows: (1) good gallbladder function, (2) stone size $<10 \mathrm{~mm}$ in diameter, (3) absence of stricture at the bottom of the bile duct, and (4) applicability of CHCs for both extrahepatic and intrahepatic stones, as well as the residual stones following surgery [28]. Only few CHCs are approved by the China Food and Drug Administration (CFDA) for treating cholelithiasis. Thirteen CHCs are presented in Table 1. We included the CHCs used in the previous systematic review wherein their efficacies were refuted [23]. Interestingly, Lysimachia christinae is included in eight out of the thirteen CHCs. It is the only ingredient in Jin Qian Cao capsule. Hence, $L$. christinae plays a role in inducing the excretion of gallstones, as demonstrated in animal models [29]. Furthermore, nbutanol is the most bioactive fraction in the L. christinae extract; it exerts antioxidant effect and endothelial protection [30]. Moreover, Deng et al. suggested that L. christinae promotes bile secretion, reduces cholesterol levels in either cystic or hepatic bile, and decreases serum cholesterol level [29]. Although complex activities of the monomers in $L$. christinae remain poorly understood, the pharmacological 
TABLE 1: Commonly used CHCs approved by CFDA.

\begin{tabular}{|c|c|}
\hline CHC Name & Ingredients \\
\hline $\begin{array}{l}\text { Changgen Anti-Lithiasis Therapy (CALT, former } \\
\text { name: Jin Qian Dan Tong Oral Liquid) }\end{array}$ & $\begin{array}{c}\text { Herba Lysimachia, Lysimachia christinae, Oriental wormwood, Polygonum } \\
\text { cuspidatum, Radix bupleuri, Dandelion, Cyperus tuber, Root of red-rooted salvia, } \\
\text { Semen cassiae torae, Smoked plum }\end{array}$ \\
\hline Jin Dan Tablet & Radix gentianae, Lysimachia christinae, Polygonum cuspidatum, Pig's bile \\
\hline Pai Shi Li Dan Granules & $\begin{array}{c}\text { Lysimachia christinae, Oriental wormwood, Radix bupleuri, Radix gentianae, Radix } \\
\text { paeoniae rubrathe (unpeeled) root of common peony, Radix curcumae, Cattail pollen, } \\
\text { Excrementum pteropi, Rheum officinale, Glauber }\end{array}$ \\
\hline Li Dan Pai Shi Tablet & $\begin{array}{c}\text { Lysimachia christinae, Oriental wormwood, Scutellaria baicalensis, Radices } \\
\text { saussureae, Radix curcumae, Rheum officinale, Gglauber, Areca catechu, Fructus } \\
\text { aurantii immaturus, Magnolia officinalis }\end{array}$ \\
\hline Dan Le Capsule & $\begin{array}{c}\text { Pig's bile, Pericarpium citri reticulatae, hawthorn, curcuma aromatica, Herba } \\
\text { Lysimachia }\end{array}$ \\
\hline Dan Shi Li Tong Tablet & $\begin{array}{l}\text { Alum, Glauber, Radix curcumae, Rhizoma sparganii,Pig's bile, Lysimachia christinae, } \\
\text { Pericarpium citri reticulatae, Frankincense, myrrh, Rheum officinale, Radix liquiritiae }\end{array}$ \\
\hline Jin Jia Pai Shi Capsule & $\begin{array}{l}\text { Rhizoma sparganii, myrrh, Radix paeoniae rubrathe (unpeeled) root of common } \\
\text { peony, Peach kernel, Chinese honeylocust fruit, Angelica dahurica, Fructus aurantii } \\
\text { immaturus, Curcuma zedoary, Pericarpium citri reticulatae viride, Frankincense, } \\
\text { Semen coicis, Radices cyathulae, Plantago seed, Magnolia officinalis, Pangolin, } \\
\text { Lysimachia christinae }\end{array}$ \\
\hline Jin Qian Cao Capsule & Lysimachia christinae \\
\hline Dan Qing Capsule & Saxifraga stolonifera, Phoenix-tail fern, Rheum officinale, Oxgall \\
\hline Dan Shi Tong Capsule & $\begin{array}{c}\text { Dandelion, Radix curcumae, Oriental wormwood, Lycopodium mongolicum, } \\
\text { linearstripe rabdosia, Rheum officinale, fructus aurantia, Radix bupleuri, Scutellaria } \\
\text { baicalensis, Goose gall }\end{array}$ \\
\hline Yi Dan Tablet & Radix curcumae, Honeysuckle, Alum, Liquorice, Glauber, Talc, Radix scrophulariae \\
\hline Dan Yi Ning Tablet & Pig's bile, Plum root \\
\hline Dan Shi Qing Tablet & $\begin{array}{l}\text { Alum, Oxgall, Sheep bile, hawthorn, Radix curcumae, Rheum officinale, Glauber, } \\
\text { clematis chinensis, Endothelium corneum gigeriae galli, Glauber }\end{array}$ \\
\hline
\end{tabular}

effects of $L$. christinae need to be investigated to understand its underlying mechanisms in cholelithiasis treatment.

\subsection{CHCs Contain L. christinae}

2.1.1. Changgen Antilithiasis Therapy (CALT). CALT is a CHC system comprising several formulas that have been used in treating cholelithiasis and kidney stones for 60 years. Jin Qian Dan Tong oral liquid, which is one of the CALT formulas, is approved by CFDA particularly for cholelithiasis treatment in China. The efficacy of CALT for cholelithiasis has been proved by several randomized controlled trials (RCTs) $[14,31$, 32]. CALT promotes the discharge of gallstones and promptly relieves the pain $[14,31,32]$. Moreover, it is beneficial for the subtype with wetness heat $[14,31]$. A clinical observation found that CALT promotes bile secretion [33]. Moreover, it ameliorates the biliary motility disorder in patients who underwent LC or ERCP [34]. Interestingly, CALT has shown better efficacy than a $\mathrm{CHC}$ without L. christinae (Dan Ning tablet) in treating cholelithiasis (Figure 1) [14]. Hence, $L$. christinae is a crucial ingredient of $\mathrm{CHCs}$ and warrants further investigations.

2.1.2. Jin Dan Tablet (JDT). Zhao et al. investigated the efficacy of JDT by comparing the combination of JDT and ursodeoxycholic acid with ursodeoxycholic acid alone. They observed that the JDT group exhibited a significantly higher efficiency rate than ursodeoxycholic acid alone $(92.5 \%$ vs. $60.0 \%)$. Moreover, JDT relieved the adverse reactions associated with ursodeoxycholic acid, and the JDT group exhibited relatively lower occurrences of palpitation, blush, and loose stool than its counterpart [35].

2.1.3. Pai Shi Li Dan Granules (PSLDG). Pai Shi Li Dan granules may ameliorate postoperative complications affecting the gallbladder wall [36]. A 2-year follow-up study found that the recurrence rate of gallstones in Pai Shi Li Dan granules group was low, indicating that these granules might be effective in preventing postoperative recurrence of gallstones [22].

2.1.4. Li Dan Pai Shi Tablet (LDPST). Zhang et al. reported that Li Dan Pai Shi tablet could reduce residual gallstones in patients with intrahepatic bile duct stones. Moreover, it helps prevent the recurrence of gallstones [19].

2.1.5. Dan Le Capsule (DLC). Dan Le capsule is a common TCM medicine used for treating cholelithiasis. Its efficacy was verified by a clinical observation with 73 cases. It can relieve symptoms caused by cholelithiasis and has a gallbladderstone-discharging effect [37]. 


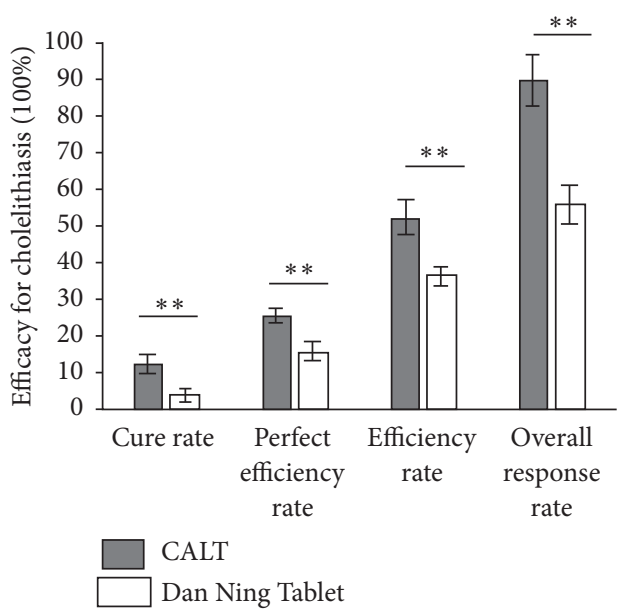

(a)

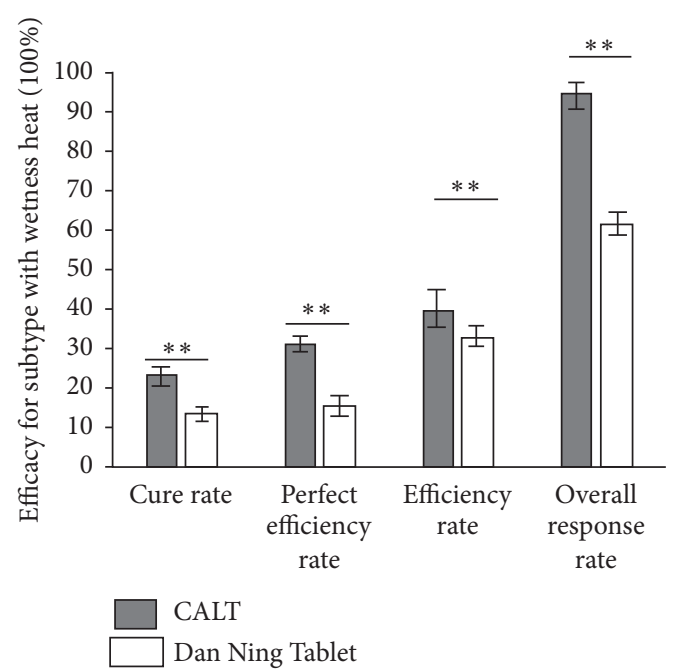

(b)

FIGURE 1: Efficacy of CALT in comparison with Dan Ning tablet. (a) CALT shows better efficacy in treating cholelithiasis than Dan Ning tablet. (b) CALT shows better efficacy in treating cholelithiasis of a subtype with witness heat than Dan Ning tablet. Data are presented as mean $\pm \mathrm{SD}, * *$ indicates $\mathrm{p}<0.01$.

2.1.6. Dan Shi Li Tong Tablet (DSLTT). He et al. conducted an RCT to verify the efficacy of Dan Shi Li Tong tablet. They found that the patients treated with such tablet achieved better cure rate $(57.78 \%$ vs. $40.00 \%, \mathrm{p}<0.05)$ and total efficiency rate $(95.56 \%$ vs. $73.33 \%, \mathrm{p}<0.05)$ than the control. This tablet can also ameliorate bilirubin metabolism and relieve the clinical symptoms [38].

\subsection{CHCs without L. christinae}

2.2.1. Dan Qing Capsule (DQC). Dan Qing capsule combined with ursodeoxycholic acid is used for treating cholelithiasis. Dan Qing capsule reduces the scores of TCM syndromes (lower score indicates better outcome), ameliorates gallbladder function, and relieves abdominal pain. Moreover, it prevents the recurrence of gallstones $[17,18]$.

2.2.2. Dan Shi Tong Capsule (DSTC). An RCT verified the efficacy of Dan Shi Tong capsule. A total of 60 patients with cholelithiasis were randomly allocated into treatment group $(\mathrm{n}=30)$ and control group $(\mathrm{n}=30)$. Study results revealed that the total efficiency rate of patients treated with Dan Shi Tong capsule was significantly higher than that of the control group (90\% vs. $73.3 \%, \mathrm{p}<0.01)$. They concluded that Dan Shi Tong capsule can remarkably relieve the clinical symptoms of cholelithiasis. Additionally, it has a good stone-discharging effect, and no adverse effect was observed [39].

2.2.3. Yi Dan Tablet (YDT). One study has proven the efficacy of Yi Dan tablet in treating cholecystitis and cholelithiasis. The efficiency rate of this tablet on cholecystitis was $>90 \%$ [40].

2.2.4. Dan Ning Tablet (DNT). Dan Ning tablet contributes to a reduced recurrence rate of gallstone formation following gallstone removal surgery (6.82\% vs. $13.64 \%)$. The gallbladder contraction index in the treatment group was better than that in the control group. The underlying recurrence-preventing mechanism might involve the elimination of biliary inflammation by regulating the intestinal flora translocation [20, 21].

2.2.5. Dan Yi Ning Tablet (DYNT). Administering Dan Yi Ning tablet for cholelithiasis resulted in $76.56 \%$ stone removal rate, with $17.97 \%$ of patients achieving complete gallstone discharge. The mechanisms might be associated with the improvement of the biliary dynamics factors [41].

2.2.6. Dan Shi Qing Tablet (DSQT). Bao et al. designed an RCT with 45 cases to investigate the efficacy of Dan Shi Qing tablet (Control group [ursodeoxycholic acid] vs. Treatment group [ursodeoxycholic acid + Dan Shi Qing tablet]). After a 2-year follow-up, they found that Dan Shi Qing tablet had a significantly lower recurrence rate than the control $(16 \%$ vs. $25 \%$ in the control group, $\mathrm{p}<0.01$ ). Hence, this tablet can reduce the postoperative recurrence rate of gallstones [42].

Aside from the abovementioned CHCs, many other $\mathrm{CHCs}$ are also commonly used for treating cholelithiasis. However, considering that no clinical trials have been reported to verify the efficacy of these CHCs (such as Jie Shi Qing capsule, Dan Shi tablet, Dan Shu Soft capsule, Shu Dan capsule, Jin Jia Pai Shi Capsule, Jin Qian Cao Capsule, Dan Kang capsule, and Li Dan Shi granule) or these medicines are not mainly used to treat cholelithiasis (such as Li Dan Xiao Yan Tablet, which is mainly used for cholecystitis), we did not introduce them in detail in accordance with the aim of this study. 


\section{Potential Mechanisms Underlying the CHC Treatments}

3.1. Amelioration of the Biliary Dynamics Factors. Changes in the biliary fluid dynamics play a crucial role in the development of gallstones [43]. Filling and emptying of the gallbladder are the results of the synergistic motility of the gallbladder, gallbladder duct, and the sphincter of Oddi. If the dynamics of the bile duct and/or gallbladder is disrupted, it may change the flow direction or produce swirls or eddies that may result in cholestasis and promote the development of cholelithiasis. As mentioned, CALT ameliorates postoperative biliary motility disorder. Indices such as the common-bile-duct pressure and the basal pressure in the sphincter of Oddi were significantly reduced by CALT administration [34]. This phenomenon might be a potential mechanism underlying the recurrence prevention. Sheng et al. reported that the 2 -week administration of Dachengqi decoction, which comprises Rheum officinale, glauber, Fructus aurantii immaturus, and Magnolia officinalis, significantly enhanced the gallbladder emptying index, indicating that the gallbladder contraction had strengthened [44]. Fang et al. revealed that emodin may ameliorate the dysfunction of gallbladder contraction and relieve cholestasis by enhancing the gallbladder contractibility. The regulation of the plasma cholecystokinin and calcium ion in the gallbladder cells may play a role in the underlying mechanisms [45]. Therefore, CHCs can strengthen gallbladder contraction [20, 21], promote bile secretion (Section 3.2), and easily discharge gallstones.

3.2. Maintenance and Protection of the Liver Function. Bile is mainly produced by the liver. When the liver is impaired, cholelithiasis may occur. Certain pathological conditions cause the liver to produce excessive cholesterol (Section 3.3) or the liver cells to synthesize superfluous bilirubin (Section 3.4), thereby resulting in the development of gallstones [46]. Reduced bile secretion causes pachycholia, possibly related to the development of gallstones. Meanwhile, the progression of cholelithiasis would adversely affect the liver function and damage the liver cells, thereby forming a vicious circle. Another example is the relationship between liver fat metabolism and cholelithiasis. An imbalance in liver fat metabolism may lead to the development of cholelithiasis [47]. Repairing liver injury and restoring the balance of liver fat metabolism would allow the recovery of cholelithiasis or prevention of gallstone formation [48]. Therefore, cholelithiasis can be ameliorated by improving the liver function. Meanwhile, CALT promotes bile secretion [33], possibly a result of an ameliorated liver function. Furthermore, according to the theories of TCM, the maintenance and protection of the liver function play a crucial role in the mechanisms of CHCs. One CHC not containing L. christinae, i.e., the Dan Ning tablet, exerts protective effects on an acutely injured liver. Administration of this tablet reduces the levels of serum alanine aminotransferase, aspartate aminotransferase, alkaline phosphatase, $\gamma$-glutamyltranspeptidase, total bilirubin, direct bilirubin, and total bile acid, as well as the hepatic myeloperoxidase, glutathione S-transferase activities, and contents of glutathione and liver lipid peroxide; in contrast, the activities of superoxide dismutase, glutathione peroxidase, and catalase were enhanced. Hence, the attenuation of the oxidative stress may be the underlying mechanism of cholelithiasis treatment using Dan Ning tablet [49].

3.3. Reduction of the Serum Cholesterol Level. Cholesterol is an essential constituent of bile. It is dissolved in the bile. When the ratio of cholesterol/phospholipids is $>1$, the existing micelles are insufficient to dissolve the whole cholesterol content. Consequently, the excessive cholesterol begins to crystallize and nucleate, indicating the mechanism of cholesterol stone formation [50]. Furthermore, the reduction of serum cholesterol level might be a result of an ameliorated liver function (Section 3.2). However, the mechanisms are complicated and multifold. Liu reported that schaftoside, which is a bioactive compound of $L$. christinae, reduces serum cholesterol level; this decrement is vital for preventing the development of cholesterol gallstones. These effects are obtained by activating the receptors of ileal liver X receptor $\alpha$ and hepatic farnesoid X receptor [51]. Another study regarding Lidan granules (Oriental wormwood, Hawthorn fruit, Rice sprout, Germinated barley, Green orange peel, Tangerine peel, Medicated leaves, Cyperus tuber, Radish seed, Caulis perillae, Turmeric root, Rhubarb, Pinellia tube, and Chinese honeylocust fruit) demonstrated that reducing cholesterol level and increasing bile phospholipid levels by downregulating the expression of inflammatory cytokines are the underlying mechanisms involved in preventing cholelithiasis [52].

3.4. Reduction of the Bilirubin Level. Bilirubin is the main pigment in bile. In certain pathological states, the bilirubin level in the bile may be enhanced and contribute to the development of bilirubin gallstones. The risk of developing gallstones is closely associated with a high bilirubin level [53]. In contrast, relieving the pathogenic factor that enhances the bilirubin (such as ameliorating the liver function) can improve and prevent bilirubin gallstones. A previous study reported the efficacy of Jinshisan in treating gallstones and discovered that this $\mathrm{CHC}$ is effective in preventing bile stone formation. The efficacy of Jinshisan treatment is highly associated with a reduction of bilirubin level in the bile [54].

3.5. Regulation of the Inflammatory Reactions. The roles of inflammatory factors in the development of gallstones are multifold and complicated. Briefly, inflammatory reactions may damage liver function, disturb biliary fluid dynamics, and cause an imbalance in bile production and secretion, thereby resulting in cholelithiasis [55]. Dan Ning tablet is efficacious in ameliorating cholelithiasis by attenuating the oxidative stress [49]. Liu et al. reported that CHCs promote bile excretion, regulate bile components, and protect the gallbladder mucosal epithelial cells. The underlying mechanisms include the suppression of cyclooxygenase- 2 and tumor necrosis factor- $\alpha$ expression via the nuclear factor-kappa $B$ signaling pathway [56]. Another study found that $\mathrm{CHCs}$ may regulate the expression of IL- 6 and EGR-1 in the hepatic tissue of a rabbit cholelithiasis model. Furthermore, chronic liver 


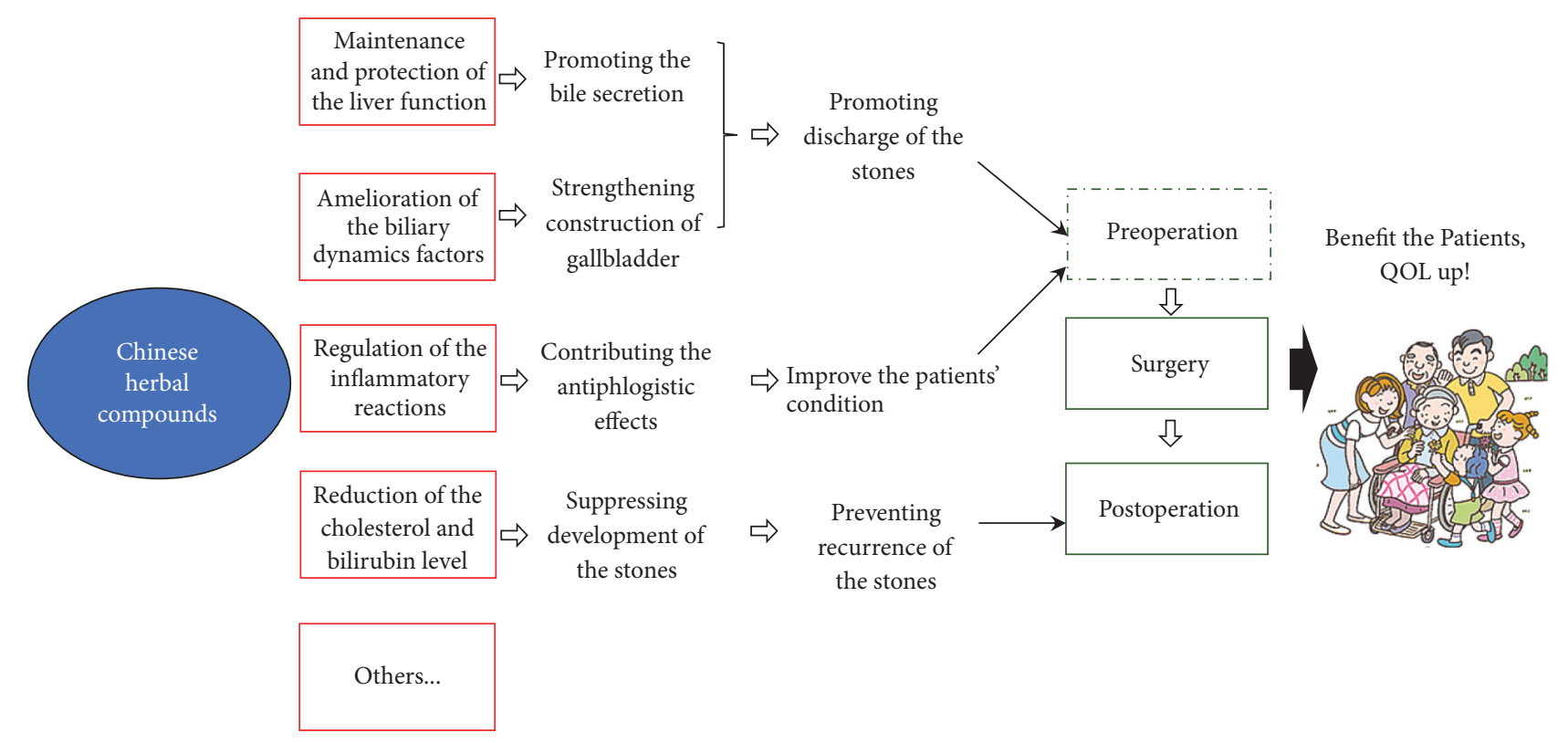

FIGURE 2: Underlying mechanisms of CHCs involved in the clinical strategy of cholelithiasis.

injury due to cholelithiasis was treated, owing to the recovery of the liver function and protection of normal hepatic cells [57]. Inflammatory factors, such as cytokines, and the inflammatory signaling pathway are crucial in the therapeutic mechanisms of $\mathrm{CHCs}$ in gallstones. Further investigations are expected to uncover the detailed inflammatory processes regarding the effects of CHCs.

\section{Concluding Remarks}

The present review summarized the underlying mechanisms of commonly used CHCs for cholelithiasis treatment. The $\mathrm{CHCs}$ included in the present review are frequently used clinically for treating cholelithiasis in China. Considering that we require the latest comprehensive information based on the contents of available studies, an updated systematic review is needed in the future.

The commonly used therapeutics, regardless of being surgical or medical, cannot circumvent the recurrence of gallstones. However, the CHCs can maintain and protect the liver function, improve biliary fluid dynamics, and regulate the inflammatory reaction. Theoretically, $\mathrm{CHCs}$ can amend the lithogenic environment either pre- or postoperatively. Hence, CHCs should prevent the recurrence of gallstones, and several studies have supported this statement [15-22]. Therefore, more well-designed RCTs are needed to provide convincing evidence regarding the effectiveness of $\mathrm{CHCs}$ in treating cholelithiasis. Once the effects of preventing the recurrence of cholelithiasis are rigorously verified, these $\mathrm{CHCs}$ can be considered as a useful adjuvant therapy for postoperative patients. CHCs can ameliorate the patients' condition perioperatively and prevent them from experiencing the recurrence of gallstones postoperatively (Figure 2).

Notably, none of the RCTs that verified the efficacy of the involved $\mathrm{CHCs}$ reported the adverse events. This finding is similar to that of our previous study [58-60]. Indeed, the lack of report regarding adverse events and followup has been a dominant problem in the TCM research [58, 59]. Several Chinese patients and TCM researchers traditionally believe that TCM therapies have no or few adverse effects [23]. However, this view is far from the truth. Our previous study reported that acupuncture cannot be regarded as a completely noninvasive therapy [61]. Recently, adverse events of CHCs have also been documented, thereby gaining considerable attention worldwide. Furthermore, the relationship between aristolochic acids and hepatocellular carcinomas has been recognized [62]. A current study found that the abuse of CHCs has been the leading cause of drug-induced liver injury (26.81\%) in China [63]. Regarding $\mathrm{CHCs}$ for treating cholelithiasis, no report is available that systematically introduces the adverse events/side effects of these medicines. However, some components of Dan Ning tablets (anthraquinone) potentially induce melanosis coli (MC) [64]. In addition, 2 out of 48 patients with MC had a history of Dan Ning tablet administration [65]. Nevertheless, the relationship between Dan Ning tablets and MC needs further investigation. However, all of these cases suggest that the adverse events of CHCs for cholelithiasis cannot be disregarded and should be seriously considered in future TCM studies. Measures such as spreading awareness concerning the adverse events of TCM in both the TCM clinicians and the general population along with establishing and improving the reporting systems are helpful to improve this problem [66]. Another important drawback of the involved studies was the lack of blinding and allocation concealment. This is a systematic problem involved in the TCM research [61]. The characteristics of TCM are "concept of wholism (holistic concept)" and "syndrome differentiation treatment (pattern differentiation)." These characteristics may cause blinding (especially the double blinding) and allocation concealment, 
which are rather difficult to be practiced in a TCM trial (the clinician must perform the individual and dynamic treatment protocol for each patent). Adoption of objective indices to prevent the results from being changed by the subjectivity from different observers (observation bias) is a good solution for this issue [61]. Hence, objectification (for both diagnosis tool and treatment) may be potentially applied in future TCM studies.

Cumulatively, TCM is characterized by the concept of wholism (holistic concept) and syndrome differentiation treatment (pattern differentiation). CHCs as treatments based on TCM can ameliorate the overall bodily function, thereby preventing the recurrence of illness. Appropriate application of CHCs would be beneficial for patients and clinicians, although their safety and efficacy need further verification.

\section{Data Availability}

The data used to support the findings of this study are included within the article.

\section{Conflicts of Interest}

The authors declare no conflicts of interest in the present study.

\section{Authors' Contributions}

Tetsuya Asakawa got the original ideas. Qiliang Chen, Yuanyuan Zhang, Shunan Li, Shujiao Chen, Xuejuan Lin, Candong $\mathrm{Li}$, and Tetsuya Asakawa searched for the literatures and extracted the information. Qiliang Chen, Yuanyuan Zhang, and Tetsuya Asakawa wrote the draft; all authors discussed and approved the last version. Candong Li and Tetsuya Asakawa supervised the study.

\section{Acknowledgments}

The study is supported by grants from the National Natural Science Foundation of China (Grant no. U1705286), grants from Fujian Province Center for Collaborative Innovation of TCM Health Management 2011 (Grant no. JG2018001), and grants from the Japanese Society for the Promotion of Science (Grant-in-Aid for Young Scientists, Type B, no. 20791025, and Grant-in-Aid for Scientific Research C, General, nos. 24592157, 15k10358 and 18k08991). The authors would like to thank Enago (www.enago.jp) for the English language review.

\section{References}

[1] T. Wilkins, E. Agabin, J. Varghese, and A. Talukder, "Gallbladder dysfunction: cholecystitis, choledocholithiasis, cholangitis, and biliary dyskinesia," Primary Care-Clinics in Office Practice, vol. 44, no. 4, pp. 575-597, 2017.

[2] F. Lammert, K. Gurusamy, C. W. Ko et al., "Gallstones," Nature Reviews Disease Primers, vol. 2, no. 1, Article ID 16025, 2016.
[3] M. H. Ahmed, M. A. Hamad, C. Routh, and V. Connolly, "Statins as potential treatment for cholesterol gallstones: An attempt to understand the underlying mechanism of actions," Expert Opinion on Pharmacotherapy, vol. 12, no. 17, pp. 26732681, 2011.

[4] M. H. Ahmed, "Ezetimbe as potential treatment for cholesterol gallstones: the need for clinical trials," World Journal of Gastroenterology, vol. 16, no. 13, pp. 1555-1557, 2010.

[5] A. N. Kalloo and S. V. Kantsevoy, "Gallstones and biliary diseases," Primary Care-Clinics in Office Practice, vol. 28, no. 3, pp. 591-606, 2001.

[6] G. Randi, S. Franceschi, and C. La Vecchia, "Gallbladder cancer worldwide: geographical distribution and risk factors," International Journal of Cancer, vol. 118, no. 7, pp. 1591-1602, 2006.

[7] M. Sato, K. Endo, A. Harada, and S. Yabuuchi, "Potential risk factors for postoperative complications and deaths after laparoscopic cholecystectomy in the elderly," Journal of Japanese Society of Gastroenterology, vol. 114, no. 9, pp. 1649-1657, 2017.

[8] J. Y. W. Lau, C.-K. Leow, T. M. K. Fung et al., "Cholecystectomy or gallbladder in situ after endoscopic sphincterotomy and bile duct stone removal in Chinese patients," Gastroenterology, vol. 130, no. 1, pp. 96-103, 2006.

[9] C. Friis, J. P. Rothman, J. Burcharth, and J. Rosenberg, "Optimal timing for laparoscopic cholecystectomy after endoscopic retrograde cholangiopancreatography: a systematic review," Scandinavian Journal of Surgery, vol. 107, no. 2, pp. 99-106, 2018.

[10] L. McHenry and G. Lehman, "Difficult bile duct stones," Current Treatment Options in Gastroenterology, vol. 9, no. 2, pp. 123-132, 2006.

[11] Y. Nakai, H. Kogure, A. Yamada, H. Isayama, and K. Koike, "Endoscopic management of bile duct stones in patients with surgically altered anatomy," Digestive Endoscopy, vol. 30, supplement 1, pp. 67-74, 2018.

[12] L. Ye, J. Liu, Y. Tang et al., "Endoscopic minimal invasive cholecystolithotomy vs laparoscopic cholecystectomy in treatment of cholecystolithiasis in China: a meta-analysis," International Journal of Surgery, vol. 13, pp. 227-238, 2015.

[13] N. M. Delzenne, P. B. Calderon, H. S. Taper, and M. B. Roberfroid, "Comparative hepatotoxicity of cholic acid, deoxycholic acid and lithocholic acid in the rat: in vivo and in vitro studies," Toxicology Letters, vol. 61, no. 2-3, pp. 291-304, 1992.

[14] S. Y. Zhang, Y. L. Jin, X. Chi, R. G. Zhou, and L. F. Yu, "Control study on therapeutic effect of Jinqian Dantong Oral Liquid and Jindan Tablet in the treatment of cholelithiasis," Chinese Journal of Integrated Traditional and Western Medicine on Digestion, vol. 11, no. 3, pp. 160-161, 2003.

[15] Z. J. Li, X. K. Wang, and X. W. Guan, "Meta-analysis of the efficacy of traditional Chinese medicine in preventing recurrence of gallstones after minimally invasive cholecystolithotomy," Modern Diagnosis \& Treatment, vol. 16, pp. 3640-3641, 2015.

[16] Y. X. Liu, J. Li, S. J. Guo, and L. X. Ye, "Efficacy of traditional Chinese medicine on preventing recurrence of gallstones after minimally invasive cholecystolithotomy with gallbladder preservation," Clinical Journal of Traditional Chinese Medicine, vol. 18, no. 4, pp. 389-390, 2006.

[17] L. Zhao, Y. D. Shi, F. Xing, and Y. Su, "Clinical observation of Danqing Capsules combined with Ursodeoxycholic Acid Capsules in treatment of cholecystolithiasis," Drugs \& Clinic, vol. 32, no. 12, pp. 2451-2452, 2017.

[18] Z. Y. Zhang, W. S. Liu, Y. Li, and S. H. Zhang, "Clinical effect of Danqing Capsules combined with Ursodeoxycholic Acid 
Capules in the treatment of stone recurrence after cholecystolithotomy," China Medical Herald, vol. 15, no. 32, pp. 121-124, 2018.

[19] B. Z. Zhang, "Long-term effect of Lidan Paishi tablets on patients with intrahepatic bile duct stones after operation," Contemporary Medicine Forum, vol. 13, no. 13, pp. 283-284, 2015.

[20] R. T. Wang, Q. A. Jia, Y. P. Mu, Z.-m. Geng, and C. Liu, "The clinical evaluation of danning tablets on regulating gut flora displacement to eliminate biliary inflammation and prevent gallstone formation," Journal of Kunming Medical University, vol. 39, no. 1, pp. 109-112, 2018.

[21] Z. Y. Liu, "Effect of danning tablet on preventing recurrence of cholecystolithiasis after complete abdominal cholecystolithotomy," Inner Mongolia Medical Journal, vol. 50, no. 2, pp. 220221, 2018.

[22] J. Q. Chen, "Effect of lidan paishi granule on long-term therapeutic effect of patients with intrahepatic cholangiolithiasis after operation," Chinese Archives of Traditional Chinese Medicine, vol. 24, no. 8, pp. 1581-1582, 2006.

[23] T. Gan, J. Chen, S. J. Jin, and Y. Wang, "Chinese medicinal herbs for cholelithiasis," Cochrane Database of Systematic Reviews, vol. 6, Article ID CD004547, 2013.

[24] M.-P. Song, "Clinical observation on frequency-changeable electroacupuncture for treatment of cholelithiasis," Chinese Acupuncture \& Moxibustion, vol. 26, no. 11, pp. 772-774, 2006.

[25] J. Zhao, Y. Yu, M. Luo, L. Li, and P. Rong, "Bi-directional regulation of acupuncture on extrahepatic biliary system: an approach in guinea pigs," Scientific Reports, vol. 7, no. 1, article no. 14066, 2017.

[26] G.-M. Wang, F.-Y. Wen, L.-X. Li, H. Zheng, Y.-H. Huang, and Y.-Q. Song, "Observation of effect on contraction function of gallbladde by acupuncture at Jianjing (GB 21)," Zhongguo Zhen Jiu, vol. 31, no. 10, pp. 910-912, 2011.

[27] C. Xuemei, T. Jiaping, and W. Ling, "Treatment of cholelithiasis by acupuncture and oral decoction," Journal of Traditional Chinese Medicine, vol. 26, no. 3, pp. 167-169, 2006.

[28] Medicine. PCoDSDoCSoITCaW, "Consensus on diagnosis and treatment of cholelithiasis with integrated traditional Chinese and Western medicine," Chinese Journal of Integrated Traditional and Western Medicine, vol. 26, no. 2, pp. 132-137, 2017.

[29] J. Deng, M. Ren, X. Dai et al., "Lysimachia christinae Hance regresses preestablished cholesterol gallstone in mice," Journal of Ethnopharmacology, vol. 166, pp. 102-108, 2015.

[30] N. H. Wu, Z. Q. Ke, S. Wu et al., "Evaluation of the antioxidant and endothelial protective effects of Lysimachia christinae Hance (Jin Qian Cao) extract fractions," BMC Complementary and Alternative Medicine, vol. 18, no. 1, p. 128, 2018.

[31] B. H. Lin, S. Q. Fang, Z. B. Cheng et al., "Comparative study of Ginqiandantong oral liquid vs Lidanpaishi tablets in treating cholelithiasis," Chinese Journal of New Drugs, vol. 11, no. 4, pp. 310-312, 2002.

[32] W. J. Zhang, Z. S. Li, W. F. XIe et al., "The effect and safety of ginqiandantong oral liquid on cholecystitis and cholelithiasis," Chinese Journal of Clinical Hepatology, vol. 19, no. 4, pp. 229-231, 2003.

[33] H. S. Zheng and Y. Lan, "Clinical observation of the postoperative efficacy of Jin Qian Dan Tong Oral Liquid in patients with cholelithiasis," Modern Journal of Integrated Traditional Chinese and Western Medicine, vol. 12, no. 22, article no. 2448, 2003.
[34] X. Bai, "Observation on effect of Jinqiandantong granules combined with nursing intervention for improving biliary motility disorder in patients after operation," China Pharmaceuticals, vol. 24, no. 10, pp. 92-93, 2015.

[35] H. N. Zhao, W. Y. Chen, and H. G. Li, "Clinical observation of jindan tablet and ursodeoxycholic acid in the treatment of gallstones," Heilongjiang Medicine Journal, vol. 15, no. 5, pp. 402403, 2002.

[36] F. Zhao and L. Q. Hu, "Long-term effect of Lidan Paishi Granule on stone recurrence after cholecystolithotomy," Chinese Journal of Rural Medicine and Pharmacy, vol. 25, no. 1, pp. 28-29, 2018.

[37] X. Lu, J. Cai, Q. Du, Z. Song, and J. Zhou, “Treatment of 73 cases of chronic cholecystitis with cholelithiasis observation of curative effect," Zhejiang Medical Journal, vol. 24, no. 12, pp. 751$752,2002$.

[38] X. He and J. Li, "Danshi litong tablets in treatment of 45 cases of biliary calculi operation," China Pharmaceuticals, vol. 24, no. 18, pp. 97-99, 2015.

[39] L. Zhong, "Observation on clinical efficacy of danshitong capsule on 60 cases of choleoithiasis associated with chronic cholecystitis," Modern Diagnosis \& Treatment, vol. 23, no. 2, pp. 88-89, 2012.

[40] B. Ye, S. Y. Luo, Z. W. Shan, J. Y. Ji, and Z. Q. Wang, “Treatment of 179 cases of acute and chronic cholecystitis and cholelithiasis with Yi dan tablet," Journal of Nanjing College of Traditional Chinese Medicine, vol. 9, no. 3, pp. 14-15, 1993.

[41] Group ZPDYNCVC, "Clinical observation of danyining in the treatment of 269 cases of cholelithiasis and biliary tract infection," Zhejiang Medical Journal, vol. 7, no. 6, p. 14, 1985.

[42] C. Bao and K. LV, "The clinical study of danshiqing tablet combine with ursodeoxycholic acid in preventing cholelithiasis for post-operation of intrahepatic bile duct stone," China Pharmaceuticals, vol. 22, no. 22, pp. 79-80, 2003.

[43] A. Ikoma, T. Ueno, K. Tanaka, A. Saisho, A. Yoshida, and A. Taira, "Cholesterol polyp of the common bile duct," American Journal of Gastroenterology, vol. 90, no. 9, pp. 1534-1535, 1995.

[44] Y. Sheng, C. H. Zong, J. L. Ni, and X. L. Wang, "Treatment of gallbladder hypomotility in patients with cholecystolithiasis by integrated traditional Chinese and Western Medicine," Chinese Journal of Integrated Traditional and Western Medicine, vol. 18, no. 8, p. 456, 1998.

[45] B.-J. Fang, J.-Y. Shen, H. Zhang, S. Zhou, C.-Z. Lyu, and Y.-Q. Xie, "Effect of emodin on mobility signal transduction system of gallbladder smooth muscle in Guinea pig with cholelithiasis," Asian Pacific Journal of Tropical Medicine, vol. 9, no. 10, pp. 10131018, 2016.

[46] J. Tanaja and J. M. Meer, "Cholelithiasis," in StatPearls, Treasure Island, Fla, USA, 2018.

[47] R. N. Appleby, J. D. Nolan, I. M. Johnston, S. S. Pattni, J. Fox, and J. R. F. Walters, "Novel associations of bile acid diarrhoea with fatty liver disease and gallstones: a cohort retrospective analysis," BMJ Open Gastroenterology, vol. 4, no. 1, Article ID e000178, 2017.

[48] V. Jaruvongvanich, A. Sanguankeo, and S. Upala, "Significant association between gallstone disease and nonalcoholic fatty liver disease: a systematic review and meta-analysis," Digestive Diseases and Sciences, vol. 61, no. 8, pp. 2389-2396, 2016.

[49] L.-L. Ding, B.-F. Zhang, W. Dou, L. Yang, C.-S. Zhan, and Z.-T. Wang, "Protective effect of Danning tablet on acute livery injury with cholestasis induced by $\alpha$-naphthylisothiocyanate in rats," Journal of Ethnopharmacology, vol. 140, no. 2, pp. 222-229, 2012. 
[50] M. Itani and T. J. Dubinsky, "Physical chemistry of bile detailed pathogenesis of cholelithiasis," Ultrasound Quarterly, vol. 33, no. 3, pp. 229-236, 2017.

[51] M. Liu, C. Liu, H. Chen et al., "Prevention of cholesterol gallstone disease by schaftoside in lithogenic diet-induced C57BL/6 mouse model," European Journal of Pharmacology, vol. 815, pp. 1-9, 2017.

[52] X. Wu, X. Liang, Y. Du et al., "Prevention of gallstones by Lidan Granule: insight into underlying mechanisms using a guinea pig model," Biomedical Reports, vol. 5, no. 1, pp. 50-56, 2016.

[53] W. Y. Au, W. C. Cheung, W. H. Hu et al., "Hyperbilirubinemia and cholelithiasis in Chinese patients with hemoglobin $\mathrm{H}$ disease," Annals of Hematology, vol. 84, no. 10, pp. 671-674, 2005.

[54] D. K. Pei, B. A. Feng, and F. L. Guan, "Clinical study on effect of jinshisan on dissolution of extra-/ intrahepatic biliary stones," Zhongguo Zhongxiyi Jiehe Zazhi, vol. 16, no. 1, pp. 18-20, 1996.

[55] J. H. Sang, N. K. Ki, J. H. Cho, J. O. Ahn, and J. G. Sunwoo, "Correlations between metabolic syndrome, serologic factors, and gallstones," Journal of Physical Therapy Science, vol. 28, no. 8, pp. 2337-2341, 2016.

[56] L. Z. Liu, S. S. Hu, Y. Qian, Z. H. Chen, and Y. B. Tian, "Study on mechanism of Danshiliuhao Decoction on treating cholecystitis of guinea pigs," Pharmacology and Clinics of Chinese Materia Medica, vol. 31, no. 4, pp. 179-183, 2015.

[57] Y. Yu, B. Wang, Q. L. Tang et al., "Dahuang lingxian granule treating cholelithiasis by down-regulating IL-6 and EGR-1," Liaoning Journal of Traditional Chinese Medicine, vol. 44, no. 7, pp. 1514-1518, 2017.

[58] F. Jiang, T. Yang, H. Yin et al., "Evidence for the use of acupuncture in treating parkinson's disease: update of information from the past 5 years, a mini review of the literature," Frontiers in Neurology, vol. 9, article no. 596, 2018.

[59] T. Asakawa and Y. Xia, "Can acupuncture treat Alzheimer's disease and other neurodegenerative disorders?" in Current Research in Acupuncture, pp. 255-301, Springer, 2013.

[60] T. Asakawa and Y. Xia, "Acupuncture treatment for Parkinson's disease," in Current Research in Acupuncture, pp. 215-253, Springer, 2013.

[61] T. Asakawa and Y. Xia, "Future research in acupuncture: better design and analysis for novel and valid findings," in Current Research in Acupuncture, pp. 687-725, Springer, 2013.

[62] A. W. Ng, S. L. Poon, M. N. Huang et al., "Aristolochic acids and their derivatives are widely implicated in liver cancers in Taiwan and throughout Asia," Science Translational Medicine, vol. 9, no. 412, 2017.

[63] T. Shen, Y. Liu, J. Shang et al., "Incidence and etiology of druginduced liver injury in mainland China," Gastroenterology, vol. 156, no. 8, pp. 2230-2241.e11, 2019.

[64] J. Chen, F. Pan, and T. Zhang, "Research progress of the relationship between anthraquinones and onset of the melanosis coli," Zhejiang Medical Journal, vol. 31, no. 7, pp. 1032-1033, 2009.

[65] Q. Zhang, F. Xu, P. Xiang, and R. Qin, "A clinical observation of 48-case melanosis coli," Chinese Journal of Digestive Endoscopy, vol. 19, no. 1, p. 50, 2002.

[66] H. Fang, X. Lin, J. Zhang et al., "Multifaceted interventions for improving spontaneous reporting of adverse drug reactions in a general hospital in China," BMC Pharmacology \& Toxicology, vol. 18, no. 1, p. 49, 2017. 


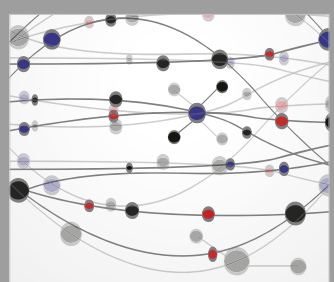

The Scientific World Journal
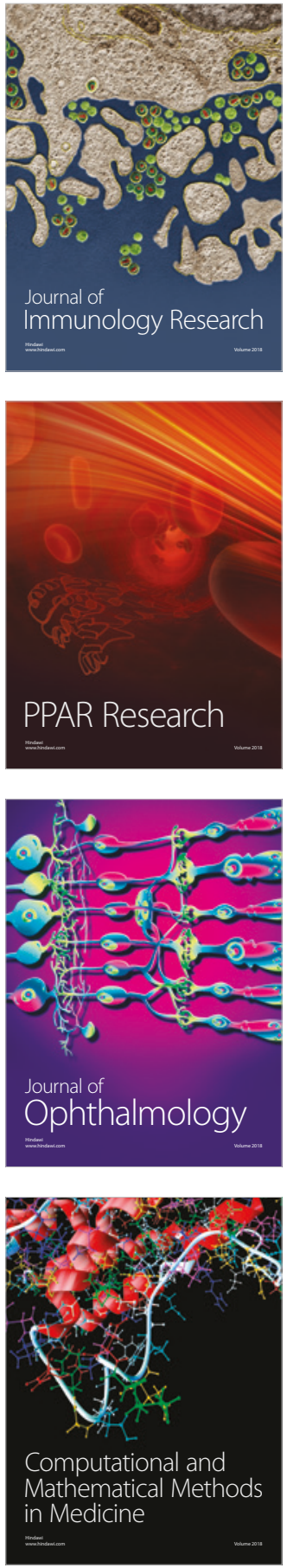

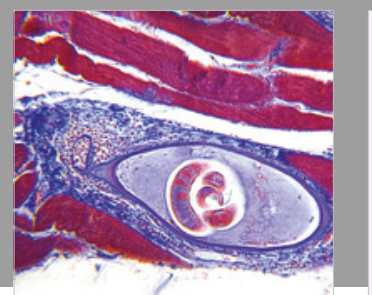

Gastroenterology Research and Practice

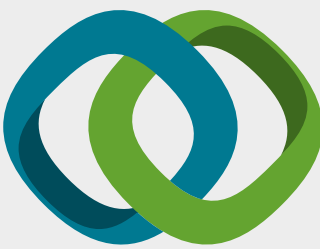

\section{Hindawi}

Submit your manuscripts at

www.hindawi.com
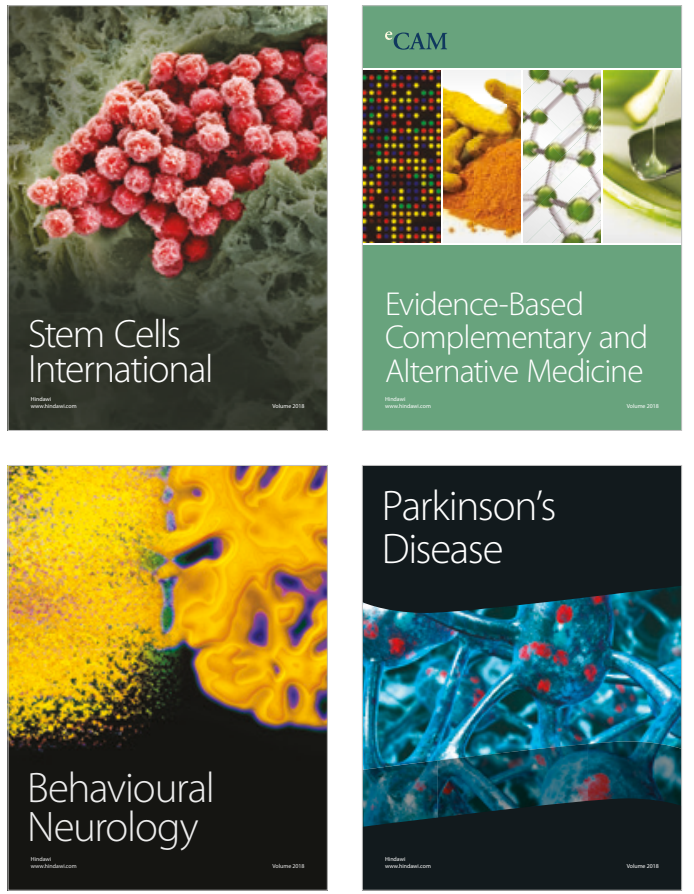

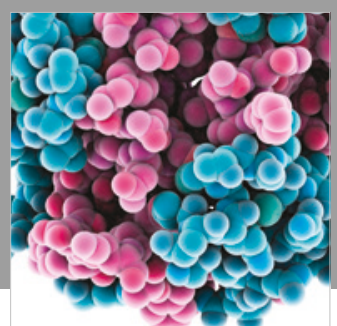

ournal of

Diabetes Research

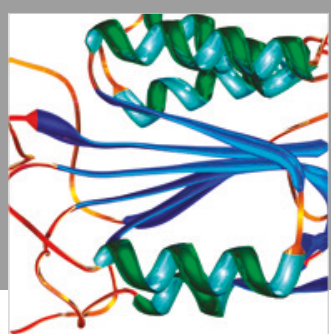

Disease Markers
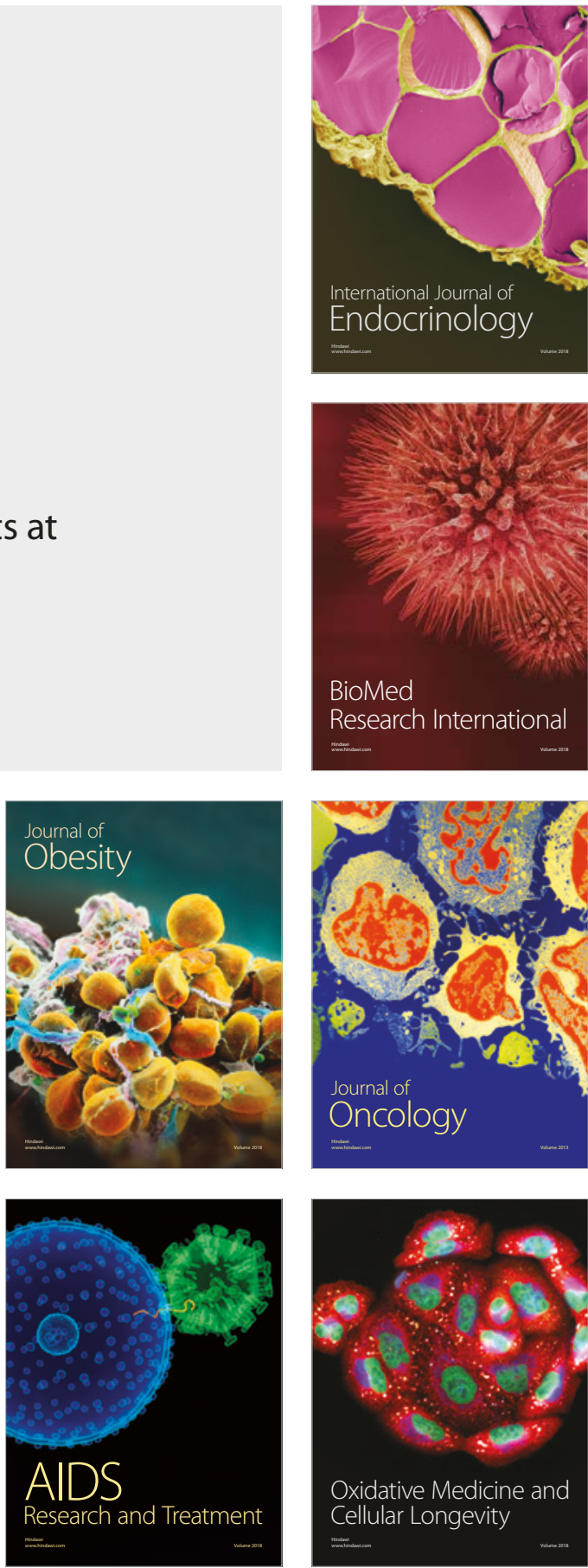